\title{
Coordination Polymers Based On Dithiophosphato/Dithiophosphonato Nickel Complexes and Linear 1,4-di(3-pyridyl)buta-1,3-diyne Ligand
}

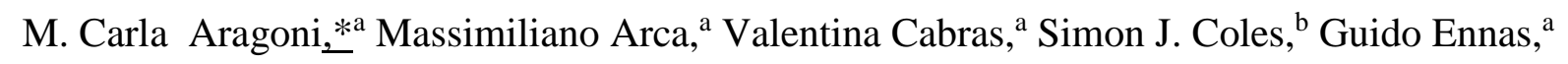
Francesco Isaia, ${ }^{\mathrm{a}}$ Romina Lai, ${ }^{\mathrm{a}}$ Vito Lippolis, ${ }^{\mathrm{a}}$ Enrico Podda. ${ }^{\mathrm{a}}$

${ }^{a}$ Dipartimento di Scienze Chimiche e Geologiche, Università degli Studi di Cagliari, S.S. 554 bivio per Sestu, 09042 Monserrato-Cagliari, Italy. ${ }^{b}$ School of Chemistry, University of Southampton, Highfield,

E-mail: aragoni@unica.it

Southampton, UK, SO17 1BJ.

\begin{abstract}
The reactions between ligand 1,4-di(3-pyridyl)buta-1,3-diyne (L) and the differently P-substituted dithiophosphato $\left[\mathrm{Ni}\left((\mathrm{RO})_{2} \mathrm{PS}_{2}\right)_{2}\right] \quad[\mathrm{R}=\mathrm{Me}(\mathbf{1})$, Et (2)] and dithiophosphonato [Ni((4$\left.\left.\left.\mathrm{MeOC}_{6} \mathrm{H}_{4}\right)(\mathrm{RO}) \mathrm{PS}_{2}\right)_{2}\right]$ [R = Me (3), Et (4)] nickel complexes have been performed, and the solid state structures of the resulting coordination polymers $[\mathbf{1} \cdot \mathbf{L}]_{\infty}-[\mathbf{4} \cdot \mathbf{L}]_{\infty}$ investigated. $\mathbf{L}$ acts as spacer between the complex units driving the formation of zig-zag polymeric chains. Inter-chain interactions involving the P-substituents have been discussed and the different supramolecular 3Dconstructs described. The $\mathbf{L}$ donor ability has been confirmed also by spectrophotometric determination of the formation constants of the species $\mathbf{1} \cdot 2 \mathbf{L}, 2 \cdot 2 \mathbf{L}$ and $\mathbf{1} \cdot 2 \mathbf{L}$, and by theoretical studies.
\end{abstract}

\section{Introduction}

In the last quarter of century, the research field of coordination polymers and Metal-Organic Frameworks (MOFs) has experienced an impressive growth and now it covers many areas of science and includes innovative materials with important applications such as gas storage, molecular sensing, and drug-delivery. ${ }^{1}$ Coordination polymers and MOFs can be prepared by combination of metal cations and organic molecules possessing two or more donor atoms positioned in divergent sites, capable of bridging metal centers to create polymeric structures. The choice of the organic (spacers) and inorganic (metal ions or coordinatively unsaturated preformed complexes) components allows designing polymeric frameworks and constitutes the basis of crystal engineering. Coordination polymers formed by covalent coordination bonds can further assemble in extended supramolecular architectures sustained by weaker interactions such as hydrogen or halogen bondings, $\pi-\pi$ interactions and other non-covalent contacts involving groups belonging to 
the organic or inorganic components of the coordination polymer. This means that by tailoring different components, it is possible to manipulate structures at the molecular level to obtain different polymeric frameworks and supramolecular architectures and hence modify the properties of the resulting materials.

In this respect, we have been developing a synthetic program based on the combination of differently P-substituted neutral organodithiophosphorous $\mathrm{Ni}^{\mathrm{II}}$ complexes, ${ }^{2}$ and a variety of polypyridyl donors, in particular 4,4'-bipyridine and its analogues, ${ }^{3}$ to assemble coordination polymers. ${ }^{4}$ This assembly process is based on the capability of the coordinatively unsaturated $\mathrm{Ni}^{\mathrm{II}}$ ion in these square-planar complexes to axially bind suitable bidentate bipyridyl-based spacers yielding 1D coordination polymers whose primary structural motif mainly depends on the features of the spacer. ${ }^{4}$ On the other hand, the substituents on the phosphorus atoms can be tailored in order to generate hydrogen bonds and/or $\pi-\pi$ interactions that sustain the connection of the polymers in extended supramolecular 3D-architectures. ${ }^{4}$ As a consequence, coordination polymers and 3D assemblies with different structures and architectures can be built up by varying either the bridging ligands or the substituents on the $\mathrm{P}$ atom of the initial $\mathrm{Ni}^{\mathrm{II}}$ complexes. We have recently demonstrated that the 3,5-di-(4-pyridyl)-1,2,4-thiadiazole spacer featuring nitrogen atoms parapositioned in the outwards pyridyl rings self-govern the geometry of the resulting supramolecular construct, leading to predictable assembly of linear or smoothly undulating chains independent of the nature of the interacting Lewis acid. ${ }^{4 d}$ On the contrary, the isomer 3,5-di-(3-pyridyl)-1,2,4thiadiazole featuring meta-positioned nitrogen atoms in the outwards pyridyl rings, allows for the existence of different supramolecular constructs ensuing from different ligand conformations deriving from the rotation of the pyridyl rings, also depending on the influence of secondary interactions involving the P-substituents. Following these results, in order to better understand how the steric information contained in the P-substituents influences the process of molecular recognition between linear coordination polymers containing spacers featuring meta-positioned nitrogen atoms in the outwards pyridyl rings, we investigated the reactivity of the ligand 1,4-di(3pyridyl)buta-1,3-diyne (L), with differently P-substituted dithiophosphonato and dithiophosphato complexes $\left[\mathrm{Ni}\left((\mathrm{MeO})_{2} \mathrm{PS}_{2}\right)_{2}\right](\mathbf{1}),\left[\mathrm{Ni}\left((\mathrm{EtO})_{2} \mathrm{PS}_{2}\right)_{2}\right](2),\left[\mathrm{Ni}(\mathrm{MeOdtp})_{2}\right]$ (3), and [Ni(EtOdtp) 2$](4)$ (Scheme 1). 


\section{Results and Discussion}

The bidentate ligand 1,4-di(3-pyridyl)buta-1,3-diyne (L) belongs to the family of bis(aryl)diacetylenes. These find roles as building blocks and are widely investigated due to the strong tendency of the diacetylene moiety to arrange into columnar systems thus leading to polymeric conjugated systems which display interesting linear and non-linear optical properties. ${ }^{5}$ The ligand $\mathbf{L}$ was first prepared and structurally characterized by Rodríguez et al. in 1997, ${ }^{6}$ and used as a linker for the preparation of several discrete supramolecular structures, ${ }^{7}$ and coordination polymers. ${ }^{8}$ Due to the presence of the $\mathrm{C}$-C single bond, different orientations are possible for the pyridyl rings, producing several ligand conformations that can be expected to produce different coordination constructs. Therefore, a Potential Energy Surface (PES) analysis was carried out on $\mathbf{L}$ at DFT level in the gas phase by rotating one pyridyl substituent through an angle $\tau$ ranging between -180 and $180^{\circ}$. This clearly shows that the donor displays an energy minimum when the pyridyl rings are coplanar and with the N-donors trans-oriented ( $\tau=180^{\circ}$, Figure S1), as found in the majority of the reported crystal structures. ${ }^{9}$ Energy maxima are found for the two conformations with mutual perpendicular pyridyl rings, ( $\tau=90$ and $270^{\circ}$, Figure S1), even if the energy difference between the conformations is almost neglegible $\left(0.25 \mathrm{kcal} \mathrm{mol}^{-1}\right)$. Moreover, as previously described, L features the occupied Kohn-Sham molecular orbitals HOMO-1, HOMO-2, and HOMO-3 (Figure S2 in ESI) localized on the negatively charged nitrogen atoms (natural charge = $0.483|\mathrm{e}|$ ) of the two pyridine moieties, thus confirming their ability to behave as donor sites (Figure $\mathrm{S} 2$ in ESI).

The reactions of $\mathbf{L}$ with nickel dithiophosphato [Ni((RO) $\left.\left.)_{2} \mathrm{PS}_{2}\right)_{2}\right]$ [R = Me (1), Et (2); Scheme 1]; and dithiophosphonato complexes [Ni(ROdtp) $)_{2}$ [MeOdtp $=\left(\mathrm{CH}_{3} \mathrm{O}\right)\left(4-\mathrm{ROC}_{6} \mathrm{H}_{4}\right) \mathrm{PS}_{2}{ }^{-}, \mathrm{R}=\mathrm{Me}(3)$, Et (4); Scheme 1] under solvothermal conditions afforded solid, crystalline compounds, which were isolated and identified by means of single crystal X-ray diffraction as coordination polymers of formula $(\mathbf{1} \cdot \mathbf{L})_{\infty},(\mathbf{2} \cdot \mathbf{L})_{\infty},(\mathbf{3} \cdot \mathbf{L})_{\infty}$, and $(\mathbf{4} \cdot \mathbf{L})_{\infty}$, respectively. Crystallographic data and selected bond lengths and angles are reported in Tables 1 and 2 respectively. Coordination polymers $(\mathbf{1} \cdot \mathbf{L})_{\infty}-$ $(\mathbf{3} \cdot \mathbf{L})_{\infty}$ are symmetric with the central nickel ion and the spacer $\mathbf{L}$ positioned on inversion centres, so that only half molecule contributes to the asymmetric unit.

Coordination polymers $(\mathbf{1} \cdot \mathbf{L})_{\infty}-(\mathbf{4} \cdot \mathbf{L})_{\infty}$ show similar coordination environments: the nickel ions display distorted octahedral geometries with iso-bidentate $\mathrm{Ni}-\mathrm{S}$ bonds with two dithiophosphoric ligands in the equatorial plane, and the spacer $\mathbf{L}$ axially bridging adjacent $\mathrm{Ni}^{\mathrm{II}}$ ions through the trans-oriented $\mathrm{N}$-pyridine donor sites to form infinite polymeric chains. The relevant bond lengths 
and angles (Table 2) are similar to those found in analogous coordination polymers. ${ }^{4}$ The structures of the coordination polymers, shown in Figure 1, share the presence of neutral zig-zag polymeric chains with very similar intrapolymer $\mathrm{Ni} \cdots \mathrm{Ni}$ distances of $13.87,13.81,13.88$, and $13.80 \AA$ for $(\mathbf{1} \cdot \mathbf{L})_{\infty},(\mathbf{2} \cdot \mathbf{L})_{\infty},(\mathbf{3} \cdot \mathbf{L})_{\infty}$, and $(\mathbf{4} \cdot \mathbf{L})_{\infty}$, respectively, in line with those found in other coordination polymers featuring $\mathbf{L}$ as the spacer. ${ }^{9}$ It is interesting to note that notwithstanding the similar primary structures of the polymers, the different nature of the P-substituents determines different interpolymeric interactions that give rise to different extended supramolecular 3D-architectures.

In the crystal packing of $(\mathbf{1} \cdot \mathbf{L})_{\infty}$, the chains run along the $b$ axis direction and are parallel to each other with metal nodes of adjacent chains shifted by $b / 2$. The P-methoxy substituents point towards the coordinated pyridine rings with a $\mathrm{C} 1-\mathrm{H} 1 \mathrm{c} \cdots \mathrm{Py}_{\text {centroid }}$ distance of $3.05 \AA$, and do not contribute to inter-chain linking. Polymeric chains interact with each other through weak hydrogen bonds involving the C5-H5 hydrogen atom of pyridine and the coordinated sulfur atom S2 ( $\boldsymbol{a}$ in Figure 2a and Table 3). These give rise to a $2 \mathrm{D}$ layered arrangement of $1 \mathrm{D}$ chains oriented in the crystallographic $a b$ plane. The layers pack along the $c$ axis direction through weak hydrogen bonds involving the $\mathrm{C} 6-\mathrm{H} 6$ hydrogen atom of pyridine and the $\mathrm{O} 2$ oxygen of the pertinent $\mathrm{MeO} \mathrm{P}-$ substituent ( $\boldsymbol{b}$ in Figure 2b and Table 3).

In the coordination polymer $(\mathbf{2} \cdot \mathbf{L})_{\infty}$ the chains pack in a parallel arrangement, interacting through weak hydrogen bonds ( $\boldsymbol{c}$ and $\boldsymbol{d}$ in Figure 3 and Table 3) involving the C7- $\mathrm{H} 7$ and C8-H8 hydrogen atoms of pyridine and the coordinate sulfur atom S2 and the $\mathrm{O} 1$ oxygen of the pertinent $\mathrm{MeO}$ P-substituent, respectively. The ethoxy P-substituents control the packing: methylene (PO) $\mathrm{CH}_{2}$ groups form an intramolecular interaction with the adjacent pyridyl rings with a C2H2b $\cdots$ Pycentroid distance of $3.49 \AA$; the terminal methyl C1 atom points towards the acetylenic triple bond of the ligand and contacts (e, Figure 3, Table 3) between the chains are observed.

The crystal structures of coordination polymers $(\mathbf{3} \cdot \mathbf{L})_{\infty}$ and $(\mathbf{4} \cdot \mathbf{L})_{\infty}$ exhibit disorder relating to the methoxyphenyl P-substituents and since they are directly involved in packing interactions a rigorous analysis of the final supramolecular architecture cannot be performed. Nevertheless, some general considerations can be drawn. The polymeric chains tend to interact with each other through weak H-bonds $\left(\boldsymbol{f}, \boldsymbol{g}, \boldsymbol{h}, \boldsymbol{i}\right.$ in Table 3) similar to those found in $(\mathbf{1} \cdot \mathbf{L})_{\infty}$ and $(\mathbf{2} \cdot \mathbf{L})_{\infty}$ as evidenced in Figures $4 \mathrm{a}$ and 5 a for $(\mathbf{3} \cdot \mathbf{L})_{\infty}$ and $(\mathbf{4} \cdot \mathbf{L})_{\infty}$, respectively. Layers of such interweaving parallel chains related by 2-fold screw axes, pack through interactions mainly involving the aromatic Psubstituents, as shown in Figure $4 \mathrm{~b}$ and $5 \mathrm{~b}$ for $(\mathbf{3} \cdot \mathbf{L})_{\infty}$ and $(\mathbf{4} \cdot \mathbf{L})_{\infty}$, respectively. It is interesting to note that the different final 3D architectures obtained starting from dithiophosphonato complexes 3 
and 4 bearing bulky aromatic P-substituents, lead to the formation of small cavities with calculated volumes of $206 \AA^{3}$ for $(\mathbf{3} \cdot \mathbf{L})_{\infty}$ which are occupied by $\mathrm{CHCl}_{3}$ solvent molecules in $(\mathbf{4} \cdot \mathbf{L})_{\infty}$ (Figure 6).

In order to investigate the solution equilibria related to the formation of coordination polymers $(\mathbf{1} \cdot \mathbf{L})_{\infty}-(\mathbf{4} \cdot \mathbf{L})_{\infty}, \mathrm{UV}$-visible spectrophotometric titrations were performed. Figure 7 shows the UVvis spectra collected during the titration of a $9.41 \cdot 10^{-3} \mathrm{M} \mathrm{CHCl}_{3}$ solution of 2 with increasing amounts of a $2.49 \cdot 10^{-2} \mathrm{M}$ solution of $\mathbf{L}$ in the same solvent using an Eppendorf electronic automatic dispenser (5-100 $\mu \mathrm{L}$ ). The starting volume of the solution, the amounts of $\mathbf{L}$ added, the final concentrations calculated for the reagents and their molar ratios are reported in Table 3 . The titration data were analyzed with $\mathrm{HypSpec}^{10}$ and two absorbing species were found to be involved in the equilibrium, recognizable as the square planar complex $\mathbf{2}$ and a $\mathbf{2} \cdot \mathbf{2} \mathbf{L}$ adduct formed through the addition of two $\mathbf{L}$ ligands to the central $\mathrm{Ni}^{\mathrm{II}}$ ion. The coordination of the second pyridyl group of $\mathbf{L}$ to another $\mathbf{2}$ moiety leads to the formation of the insoluble coordination polymer that precipitates from the solution as a green powder. The data were fitted using a 1:2 model and the formation constant for the equilibrium: $\mathbf{2}+2 \mathbf{L} \leftrightarrows 2 \cdot 2 \mathbf{L}$ was calculated by least squares methods $\left(\log \beta_{\text {eq }}=\right.$ $4.16 \pm 0.01)$.

A comparison of the calculated formation constant with those previously reported for the adduct formation between 3 and pyridine (Py) and $o-, m$-, $p$-aminopyridine $\left(o-, m-, p-\mathrm{NH}_{2} \mathrm{Py}\right)^{11}$ support the identity of the involved species as the ligand $\mathbf{L}$, the square planar complex $\mathbf{2}$ and the octahedral adduct $\mathbf{2} \cdot \mathbf{L}_{2}$. In Figure 8 the calculated distribution curves for the two absorbing species $\mathbf{2}$ and $\mathbf{2} \cdot \mathbf{2} \mathbf{L}$ involved in the equilibrium are reported. The experimental data for titrations of $\mathbf{1}$ and $\mathbf{3}$ with $\mathbf{L}$ are reported in the ESI, whilst the titration of complex $\mathbf{4}$ failed due to incipient precipitation of the final product. Table 4 summarizes the formation constants calculated for $\mathbf{1} \cdot 2 \mathbf{L}, 2 \cdot 2 \mathbf{L}$, and $3 \cdot 2 \mathbf{L}$, along with those previously determined for adducts $3 \cdot \mathrm{Py}_{2}, 3 \cdot\left(o-\mathrm{NH}_{2} \mathrm{Py}\right)_{2}, \mathbf{3} \cdot\left(m-\mathrm{NH}_{2} \mathrm{Py}\right)_{2}$, and $\mathbf{3} \cdot(p-$ $\left.\mathrm{NH}_{2} \mathrm{Py}\right)_{2}$, reported for comparison. On passing from $\mathbf{1} \cdot 2 \mathbf{L}$ to $2 \cdot 2 \mathbf{L}$ a decrease in the $\beta$ value is observed testifying for the decreased Lewis acidity of the complex on passing from methoxy to ethoxy P-substituent. A similar decrease is observed on passing from two to one methoxy substituents, in $\mathbf{1} \cdot 2 \mathbf{L}$ and $\mathbf{3} \cdot 2 \mathbf{L}$, respectively. The obtained results suggest that the initial step of the formation of the coordination polymers $(\mathbf{1} \cdot \mathbf{L})_{\infty}-(\mathbf{4} \cdot \mathbf{L})_{\infty}$ is the coordination of two $\mathbf{L}$ molecules to the same $\mathrm{Ni}^{\mathrm{II}}$ ion with formation of an 1:2 octahedral complex bearing two monocoordinated $\mathbf{L}$ donors. The subsequent coordination of the free pyridyl rings of the bonded ligands to $\mathrm{Ni}^{\mathrm{II}}$ ions of additional dithiophosphonato or dithiophosphato complexes, leads to the formation of the relative $1: 1$ coordination polymer. 


\section{Conclusions}

The reaction of $\mathbf{L}$ with the differently P-substituted dithiophosphonato and dithiophosphato complexes 1-4 yielded the corresponding coordination polymers $(\mathbf{1} \cdot \mathbf{L})_{\infty}-(\mathbf{4} \cdot \mathbf{L})_{\infty}$ featuring similar monodimensional zig-zag assemblies where $\mathbf{L}$ acts as spacer between adjacent $\mathrm{Ni}^{\mathrm{II}}$ nodes. Notwithstanding different orientations of the pyridyl rings are allowed for $\mathbf{L}$, polymers $(\mathbf{1} \cdot \mathbf{L})_{\infty}-$ $(\mathbf{4} \cdot \mathbf{L})_{\infty}$ all feature $\mathbf{L}$ in the more stable planar conformation with trans-oriented N-donors. On the contrary, the final supramolecular constructs are different and depend on the nature of the Psubstituents: $O$-alkyl substituents in polymers $(\mathbf{1} \cdot \mathbf{L})_{\infty}$ and $(\mathbf{2} \cdot \mathbf{L})_{\infty}$ lead to more dense final packings, whilst cavities have been established in the final construct of $(\mathbf{3} \cdot \mathbf{L})_{\infty}$ and $(\mathbf{4} \cdot \mathbf{L})_{\infty}$ when bulky aromatic substituents are present at the P-atom. These results indicate that $\mathbf{L}$ can be used as spacer for the predictable assembly of zig-zag chains even in the presence of different secondary interactions involving the polymeric chains and leading to different supramolecular 3D-constructs. The $\mathbf{L}$ donor ability was also confirmed by spectrophotometric determination of the formation constants of the species $\mathbf{1} \cdot 2 \mathbf{L}, \mathbf{2} \cdot 2 \mathbf{L}$, and $\mathbf{3} \cdot 2 \mathbf{L}$, suggesting the initial formation of discrete $1: 2$ complex to ligand complexes.

\section{Experimental}

\section{Materials and Methods}

Starting materials and solvents were purchased from commercial sources and when necessary the solvents have been distilled according to standard literature techniques. Melting point measurements were carried in capillaries, using electro thermal melting point apparatus $\left(0-250^{\circ}\right.$ Celsius range). ${ }^{1} \mathrm{H}-\mathrm{NMR}$ (400-MHz) for ligand $\mathbf{L}$ in $\mathrm{CDCl}_{3}$, was recorded at $25{ }^{\circ} \mathrm{C}$ on a Varian INOVAX-500 spectrometer. Chemical shifts for ${ }^{1} \mathrm{H}-\mathrm{NMR}$ are reported in parts per million (ppm), calibrated to the residual solvent peak set, with coupling constants J reported in Hertz (Hz). Infrared (IR) spectra were recorded on a Thermo Nicolet 5700 FTXIR spectrophotometer using KBr pellets and reported in wavenumbers $\left(\mathrm{cm}^{-1}\right)$. UV-vis spectra were recorded with an UV spectrophotometer Thermo Nicolet Evolution (190-1100 nm) in a range of 400-800 nm.

Single-crystal X-ray diffraction data was collected at $100 \mathrm{~K}$ on a Rigaku AFC12 goniometer equipped with an enhanced sensitivity (HG) Saturn 724+ detector mounted at the window of an FRE+ Superbright Mo-K $\alpha$ rotating anode generator $(\lambda=0.71075 \AA)$ with HF or VHF varimax optics. ${ }^{12}$ Unit cell parameters were refined against all data and an empircal absorption correction applied in Crystal Clear. ${ }^{13}$ All structures were solved by direct methods using SHELXS-2013 ${ }^{14}$ and refined on $F_{O}{ }^{2}$ by SHELXL-2013 ${ }^{14}$ in the OLEX2 environment. ${ }^{15}$ All hydrogen atoms were added in 
calculated positions and refined in riding mode on the parent atom. For $(\mathbf{3} \cdot \mathbf{L})_{\infty}$ and $\left.\mathbf{( 4 \cdot} \cdot \mathbf{L}\right)_{\infty}$ disordered phenyl ring modelled over two positions however greater rotation seen and likely to be in more than two positions. Further modelling of disorder did not improve model. SMBTX used to model unknown solvent, likely to be $\mathrm{MeOH}$ and/or $\mathrm{CHCl}_{3}$. Structures have been deposited with the Cambridge Crystallographic Data Centre with deposition numbers CCDC1563610-CCDC1563613 for $(\mathbf{1} \cdot \mathbf{L})_{\infty},(\mathbf{2} \cdot \mathbf{L})_{\infty},(3 \cdot \mathbf{L})_{\infty}$ and $(\mathbf{4} \cdot \mathbf{L})_{\infty}$ respectively.

\section{Theoretical Calculations}

Quantum chemical calculations based on the Density Functional Theory (DFT) ${ }^{16}$ level were carried out on $\mathbf{L}$ with the Gaussian $09^{17}$ by adopting the mPW1PW ${ }^{18}$ functional and using the Def2SVP ${ }^{19}$ split-valence plus polarisation (pVDZ) all-electron basis sets (BSs) for all atomic species. A potential energy surface was carried out by rotating one of the pyridine rings by an angle $\tau\left(-180.0^{\circ}\right.$ $\leq \tau \leq 180.0^{\circ} ; \Delta \tau=5.0^{\circ}$ ). The programmes GaussView 5.0.8 and Molden 5.2 were used to investigate the charge distributions and MO shapes. ${ }^{20}$

\section{Syntheses}

\section{1,4-di(3-pyridyl)buta-1,3-diyne (L)}

The ligand 1,4-di(3-pyridyl)buta-1,3-diyne (L) was synthesized optimizing the literature methods ${ }^{6}$ by using small quantity of bis(triphenylphosphine)palladium (II) dichloride catalyst thus increasing the yield and slowing down the reaction time. A mixture of 3-ethynylpyridine (0.996 g, 9.65 mmol), copper iodide (0.056 g, $0.30 \mathrm{mmol})$, bis(triphenylphosphine)palladium(II) dichloride (0.133 g $0.19 \mathrm{mmol})$ and diethylamine $(50 \mathrm{~mL})$ was stirred at reflux temperature for 5 hours. The mixture was then cooled at room temperature and filtered under reduced pressure, washed with brine a saturated $\mathrm{NH}_{4} \mathrm{Cl}$ solution $(20 \mathrm{~mL})$ and extracted with ethyl acetate $(3 \times 15 \mathrm{~mL})$ dried with $\mathrm{Na}_{2} \mathrm{SO}_{4}$ and the solvent evaporated under reduced pressure. The crystallization of the yellow solid from dichloromethane-ethanol yielded $\mathbf{L}$ as colorless crystals (0.603 g, 2,95 mmol, 61 \% yield). M.p 148 ${ }^{\circ} \mathrm{C}$. Spectral data: ${ }^{1} \mathrm{H}$ NMR (500 MHz CDCl $\left.3298 \mathrm{~K}\right) \delta=8.77(\mathrm{~d}, 2 \mathrm{H}),, \delta=8.61(\mathrm{dd}), \delta=7.82(\mathrm{dt})$, $\delta=7.30$ (t). FT-IR (KBr, 4000-400 cm $\left.{ }^{-1}\right) \tilde{v}=2128(\mathrm{w}), 1577(\mathrm{~m}), 1473(\mathrm{~m}), 1412(\mathrm{~s}), 1188(\mathrm{~m})$, 1022 (s), 802 (s), 698 (s), 627 (m), $513(\mathrm{w})$. Fluorescence $\left(\mathrm{CHCl}_{3} ; \lambda\right.$ ex $=\lambda_{\text {ex }}=263$ nm.; slit $\left.=5 \mathrm{x} 5\right)$ : $\lambda \mathrm{em}=357 \mathrm{~nm}$ and $369 \mathrm{~nm}, \mathrm{M}=1.14 \cdot 10^{-6}$

\section{$\left.\left[\left((\mathrm{MeO})_{2} \mathrm{PS}_{2}\right)_{2} \mathrm{Ni} \cdot \mathbf{L}\right)\right]_{\infty},(\mathbf{1} \cdot \mathbf{L})_{\infty}$}

(29.6 mg, $0.08 \mathrm{mmol})$ of [((MeO) $\left.\left.)_{2} \mathrm{PS}_{2}\right)_{2} \mathrm{Ni}\right](\mathbf{1})$ and $(30.5 \mathrm{mg}, 0.15 \mathrm{mmol})$ of $\mathbf{L}$, have been reacted at $130{ }^{\circ} \mathrm{C}$ in high pressure Aldrich tube in $15 \mathrm{~mL}$ of $\mathrm{MeOH}$ and $15 \mathrm{~mL}$ of $\mathrm{CHCl}_{3}$ (amylene stabilized). After complete dissolving of the reagents, the reaction mixture was transferred in a vial, and slowly cooled at room temperature. After two weeks green needles crystals of $(\mathbf{1} \cdot \mathbf{L})_{\infty}$, suitables for X-ray analysis have been obtained (10 $\mathrm{mg}, 0.13 \cdot 10^{-3} \mathrm{mmol}, 55 \%$ yield). M.p $>250^{\circ} \mathrm{C}(\mathrm{m})$ not detectable. 
FT-IR (KBr, 4000-400 cm-1): 3415 m, 2937 w, 1638 w, 1478 w 1175 w, 1016 s, 798 s, 695 w, 673 w, $659 \mathrm{w}$.

\section{$\left.\left[\left((\mathrm{EtO})_{2} \mathrm{PS}_{2}\right)_{2} \mathrm{Ni} \cdot \mathrm{L}\right)\right]_{\infty},(2 \cdot \mathrm{L})_{\infty}$}

(56.8 mg, $0.13 \mathrm{mmol})$ of [((EtO) $\left.\left.)_{2} \mathrm{PS}_{2}\right)_{2} \mathrm{Ni}\right](2)$ and $(51.0 \mathrm{mg}, 0.25 \mathrm{mmol})$ of $\mathbf{L}$, were reacted at 130 ${ }^{\circ} \mathrm{C}$ in a high pressure Aldrich tube in $15 \mathrm{~mL}$ of $\mathrm{EtOH}$ and $15 \mathrm{ml}$ of $\mathrm{CHCl}_{3}$. After complete dissolving of the reagents, the reaction mixture was transferred in a small vial and slowly cooled at room temperature. After two weeks was obtained very few green palette crystals of $(\mathbf{2} \cdot \mathbf{L})_{\infty}$ suitable for X-ray analysis. M.p=170 ${ }^{\circ}$ C. FT-IR (KBr, 4000-400 cm $\left.\mathrm{cm}^{-1}\right)$ : $3441 \mathrm{~m}, 2973 \mathrm{~m}, 2925 \mathrm{w}, 1738 \mathrm{w}$ 1618 w, 1440w, 1384 vw, 1101 m, 1023 s, 801 m 644 m 538 w, cm

\section{$\left.\left[\left(\left(\mathrm{MeO}-\mathrm{C}_{6} \mathrm{H}_{4}\right)(\mathrm{MeO}) \mathrm{PS}_{2}\right)_{2} \mathrm{Ni} \cdot \mathrm{L}\right)\right]_{\infty},(3 \cdot \mathrm{L})_{\infty}$}

(96.0 mg, $0.18 \mathrm{mmol})$ of [((MeO) $\left.\left.)_{2} \mathrm{PS}_{2}\right)_{2} \mathrm{Ni}\right]$ (3) and (70 mg; $\left.0.35 \mathrm{mmol}\right)$ of 1,4-di(3-pyridyl)buta1,3-diyne ( $\mathbf{L})$, have been reacted at $50{ }^{\circ} \mathrm{C}$ in a flask with $15 \mathrm{~mL}$ of $\mathrm{MeOH}$ and $15 \mathrm{~mL}$ of $\mathrm{CHCl}_{3}$ (amylene stabilized). After complete dissolving of the reagents, the reaction mixture was slowly cooled at room temperature. After twenty-four hours a green microcrystalline powder of (3.L1) $)_{\infty}$ is precipitated from solution (45 mg; $0.48 \mathrm{mmol}$; 89\%yeld). M.p.: $180{ }^{\circ} \mathrm{C}(\mathrm{m})$. Green crystals suitable for X-ray analysis were obtained by preparing a solution of 3 (20 mg; $0.458 \mathrm{mmol})$ in $5 \mathrm{~mL}$ of $\mathrm{CHCl}_{3}$ (amylene stabilized) in a small vial which was then introduced into a bigger one containing solution of $\mathbf{L}$ (17.5 mg; $0.08 \mathrm{mmol}$ ) in $10 \mathrm{~mL}$ of $\mathrm{MeOH}$, and left to stand at room temperature for a week. FT-IR (KBr, 4000-400 cm²): 1598 vs, 1568 w, 1531 vw, 1500 s, 1455 w, 1440 w, 1389 w, 1297 ms, 1259 s, 1216 mw, 1178 mw, 1114 vs, 1064 w, 1029 vs, 949 s, 830 w, 810 mw, 646 s, 628 mw, 545 ms, 521 w, 500 vw, 472 mw, 438 vs, 383 vs, 383 s, 326 vw, 301 w, 287 vw, 235 s, 199 mw, $167 \mathrm{vs}, 112 \mathrm{~ms}, 102 \mathrm{~s} \mathrm{~cm}^{-1}$.

\section{$\left.\left.\left[\left(\left(\mathrm{MeO}-\mathrm{C}_{6} \mathrm{H}_{4}\right)(\mathrm{EtO})\right) \mathrm{PS}_{2}\right)_{2} \mathrm{Ni} \cdot \mathrm{L}\right)\right]_{\infty},(4 \cdot \mathrm{L})_{\infty}$}

(35.6 mg, $0.65 \mathrm{mmol}$ ) of [((EtO) $\left.\left.)_{2} \mathrm{PS}_{2}\right)_{2} \mathrm{Ni}\right](4)$ and (25.1 mg, $0.12 \mathrm{mmol}$ ) of $\mathbf{L}$, have been reacted at $50{ }^{\circ} \mathrm{C}$ in a flask with $13 \mathrm{~mL}$ of $\mathrm{EtOH}$ and $15 \mathrm{~mL}$ of $\mathrm{CHCl}_{3}$ (amylene stabilized). After complete dissolving of the reagents, the reaction mixture was slowly cooled at room temperature. After twenty-four hours a green microcrystalline powder of $(\mathbf{4} \cdot \mathbf{L})_{\infty}$ was filtered from solution (60 mg; $0.06 \cdot 10^{-3} \mathrm{mmol} ; 32 \%$ yield) M.p: $180{ }^{\circ} \mathrm{C}(\mathrm{m})$. Green crystals suitable for X-ray analysis have been obtained by reacting in Aldrich high-pressure tube the same quantity of reagents and solvents. After complete reagent dissolving, the reaction mixture was transferred in a vial and slowly cooled at room temperature diffusion. FT-IR (KBr, $\left.{ }^{-1} 4000-400 \mathrm{~cm}^{-1}\right)$ : $3445 \mathrm{w}, 2933 \mathrm{w}, 2831 \mathrm{w}, 1500 \mathrm{~s}, 1567$ w, 1499 m, 1473 m, 1461 m, 1295 m, 1032 s, 801 m, 776 m, 692 w, 659 w, 545 m, 520 w, 439 m, $\mathrm{cm}^{-1}$. 
${ }^{1}$ (a) Ricco, R.; Pfeiffer, C.; Sumida, K.; Sumby, C.J.; Falcaro, P.; Furukawa, S.; Champness, N.R.; Doonan, C.J. CrystEngComm 2016, 18, 6532-6542. (b) Li, B.; Wen, H.-M.; Cui, Y.; Zhou W.; Qian G.; Chen, B. Adv. Mater. 2016, 28, 8819-8860.

2 (a) Arca, M.; Cornia, A.; Devillanova, F. A.; Fabretti, A. C.; Isaia, F.; Lippolis, V.; Verani, G. Inorg. Chim. Acta 1997, 262, 81-84. (b) Fernando, Q.; Green, C.D. J.Inorg.Nucl.Chem. 1967, 29, 647-654. (c) Haiduc, I. Handbook of Chalcogen Chemistry, Devillanova, F. A. Ed., Royal Society of Chemistry 2006, 593-643.

${ }^{3}$ Among the ligands most commonly employed as spacers the choice of using 4,4'-bipyridine and its analogues is due to their versatility. In fact, by introducing different groups between the two pyridyl rings a wide variety of either linear or bent, rigid or flexible spacers are available. See for example the feature article Biradha, K.; Sarkar, M.; Rajput, L. Chem. Commun. 2006, 4169-4179.

${ }^{4}$ (a) Aragoni, M. C.; Arca, M.; Champness, N. R.; Chernikov, A. V.; Devillanova, F. A.; Isaia, F.; Lippolis, V.; Oxtoby, N. S.; Verani, G.; Vatsadze, S. Z.; Wilson, C. Eur. J. Inorg. Chem. 2004, 10, 2008-2012. (b) Aragoni, M. C.; Arca, M.; Champness, N. R.; De Pasquale, M.; Devillanova, F. A.; Isaia, F.; Lippolis, V.; Oxtoby, N. S.; Wilson, C. CrystEngComm 2005, 7(60), 363-369. (c) Crespo Alonso, M.; Arca, M.; Isaia, F.; Lai, R.; Lippolis, V.; Callear, S. K.; Caricato, M.; Pasini, D.; Coles, S. J.; Aragoni, M. C. CrystEngComm 2014, 16, 8582-8590. (d) Aragoni, M. C.; Arca, M.; Coles, S. J.; Crespo Alonso, M.; Coles S. (née Huth); Davies, R. P.; Hursthouse, M. B.; Isaia, F.; Lai, R.; Lippolis, V. CrystEngComm 2016, 18, 5620-5629.

${ }^{5}$ Chen, X.; Zhou, G.; Pengand, X.; Yoon, J. Chem. Soc. Rev. 2012, 41, 4610- 4630.

${ }^{6}$ Rodríguez, J.G.; Martín-Villamil, R.; Cano, F.H.; Fonseca, I. J. Chem. Soc., Perkin Trans. 1997, 1, 709714.

${ }^{7}$ (a) Vajpayee, V.; Song, Y.H.; Jung, Y.J.; Kang, S.C.; Kim, H.; Kim, I.S.; Wang, M.; Cook, T.R.; Stang, P.J.; Chi, K.-W. Dalton Trans. 2012, 41, 3046-3052. (b) Chi, K.-W.; Addicott, C.; Arif, A.M.; Das, N.; Stang, P.J. J. Org. Chem. 2003, 68 (25), 9798-9801.

${ }^{8}$ (a) Zaman, Md.B.; Udachin, Md.Akhtaruzzaman, Y.Yamashita, J.A.Ripmeester, Chem. Commun., 2002,0, 2322-2323. (b) Md.B.Zaman, K.; Udachin, K.A.; Ripmeester, J. CrystEngComm, 2002, 4, 613-617. (c) Kilpin, K.J.; Gower, M.L.; Telfer, S.G.; Jameson, G.B.; Crowley, J.D. Inorg. Chem., 2011, 50 (3), pp 1123-1134.

${ }^{9}$ A search in the CSD (V.538, 2017) showed only 6 structures of coordination polymers containing $\mathbf{L}$ as the spacer, with torsion angles $\tau$ ranging from $178.8-180^{\circ}$, with the exceptions of silver coordination polymer $\left\{\left[\mathrm{Ag} \cdot \mathrm{L} \cdot \mathrm{CH}_{3} \mathrm{CN}\right] \mathrm{SbF}_{6} \cdot \mathrm{CH}_{3} \mathrm{CN}\right\}_{\infty}$ (CDS code OSAROQ, ref 8c), featuring $\tau \square=170.9^{\circ}$. When incorporated in discrete supramolecular assemblies, $\mathbf{L}$ deviates from linearity $\tau=-144.3$ and $86.3^{\circ}$ (refs $7 \mathrm{a}$ and $7 \mathrm{~b}$, respectively).

10 (a) P. Gans, A. Sabatini and A. Vacca, Talanta (1996), 43, 1739-1753. (b) P. Gans, A. Sabatini and A. Vacca, Annali di Chimica (1999), 89, 45-49.

${ }^{11}$ Aragoni, M. C.; Arca, M.; Demartin, F.; Devillanova, F. A.; Graiff, C.; Isaia, F.; Lippolis, V.; Tiripicchio, A.; Verani, G. J. Chem. Soc., Dalton Trans. 2001, 2671-2677.

${ }^{12}$ Coles, S. J., Gale, P. A. Chem. Sci., 2012, 3, 683-689.

${ }^{13}$ CrystalClear-SM Expert 3.1 (Rigaku, 2012).

${ }^{14}$ Sheldrick, G. Acta Cryst., 2008, A64, 112-122.

${ }^{15}$ Dolomanov, O.V., Bourhis, L.J., Gildea, R.J., Howard, J.A.K., Puschmann, H., J. Appl. Cryst., 2009, 42, 339-341.

${ }^{16}$ C. J. Cramer, in Essentials of Computational Chemistry, Chapter 8, $2^{\text {nd }}$ Ed., Wiley, Chechester, England, 2004.

${ }^{17}$ Gaussian 09, Revision A.1, M. J. Frisch, G. W. Trucks, H. B. Schlegel, G. E. Scuseria, M. A. Robb, J. R. Cheeseman, G. Scalmani, V. Barone, B. Mennucci, G. A. Petersson, H. Nakatsuji, M. Caricato, X. Li, H. P. Hratchian, A. F. Izmaylov, J. Bloino, G. Zheng, J. L. Sonnenberg, M. Hada, M. Ehara, K. Toyota, R. Fukuda, J. Hasegawa, M. Ishida, T. Nakajima, Y. Honda, O. Kitao, H. Nakai, T. Vreven, J. A. Montgomery, Jr., J. E. Peralta, F. Ogliaro, M. Bearpark, J. J. Heyd, E. Brothers, K. N. Kudin, V. N. Staroverov, R. Kobayashi, J. Normand, K. Raghavachari, A. Rendell, J. C. Burant, S. S. Iyengar, J. Tomasi, M. Cossi, N. Rega, J. M. Millam, M. Klene, J. E. Knox, J. B. Cross, V. Bakken, C. Adamo, J. Jaramillo, R. Gomperts, R. E. Stratmann, O. Yazyev, A. J. Austin, R. Cammi, C. Pomelli, J. W. Ochterski, R. L. Martin, K. Morokuma, 
V. G. Zakrzewski, G. A. Voth, P. Salvador, J. J. Dannenberg, S. Dapprich, A. D. Daniels, Ö. Farkas, J. B. Foresman, J. V. Ortiz, J. Cioslowski, and D. J. Fox, Gaussian, Inc., Wallingford CT, 2009.

${ }^{18}$ C. Adamo, V. Barone J. Chem. Phys. 1998, 108, 664-675.

${ }^{19}$ (a) F. Weigend and R. Ahlrichs, Phys. Chem. Chem. Phys. 2005, 7, 3297-3305. (b) F. Weigend, Phys. Chem. Chem. Phys. 2006, 8, 1057-1065

${ }^{20}$ (a) G. Schaftenaar and J. H. Noordik, J. Comput.-Aided Mol. Des. 2000, 14, 123-134; (b) R. Dennington, T. Keith and J. Millan, GaussView, Ver. 5.08, Semichem Inc., Shawee Mission Ks, 2009. 